\title{
Inhibition of the cutaneous eyeblink reflex by unilateral and bilateral acoustic input: The persistence of contralateral antagonism in auditory processing
}

\author{
JAMES R. ISON \\ University of Rochester, Rochester, New York \\ and \\ LINDA A. PINCKNEY \\ Nazareth College, Rochester, New York
}

\begin{abstract}
The eyeblink reflex elicited by a cutaneous stimulus is inhibited by weak auditory stimuli that are heard just before the blink. It has been shown that monaural prestimuli produce more reflex depression than binaural prestimuli do, suggesting that reflex modification is sensitive to the outcome of antagonistic connections between contralateral auditory inputs. We examined the time course of this antagonism by giving unilateral versus bilateral pairs of noise pips $100 \mathrm{msec}$ before the reflex eyeblink, with the noise pips separated by $0,1,4$, or $8 \mathrm{msec}$. Unilateral stimuli were more effective in every condition, but their advantage diminished with increased delay between the two components. The extended bilateral and unilateral trends of increasing reflex depression with increased delay meet at about $15 \mathrm{msec}$; if this extrapolation is valid, $15 \mathrm{msec}$ represents the upper limit on this system's retention of the location of a brief noise impulse. The rate of convergence of the two temporal functions reflects the decay of the antagonistic effect of one noise on its contralateral counterpart.
\end{abstract}

The human eyeblink is depressed by weak perturbations in sensory input that occur just before the stimulus that elicits the reflex (e.g., see Graham, Putnam, \& Leavitt, 1975; Hilgard, 1933; Krauter, Leonard, \& Ison, 1973). This phenomenon is not restricted to the human eyeblink, but has been seen in many species in which reflexes are elicited by abrupt sensory input (see Ison \& Hoffman, 1983). Response depression is not modality-specificfor example, the blink reflex can be elicited by auditory or by cutaneous stimulation and can be inhibited variously by light flashes (Reiter \& Ison, 1977), by changes in auditory input of various types (Cranney \& Cohen, 1985; Ison \& Pinckney, 1983), or by cutaneous stimulation (Ison et al., 1986). Reflex inhibition is not affected by variation in the intensity of the eliciting stimulus, which does, however, influence reflex amplitudes, so that in general more intense stimuli elicit more vigorous responses (Hoffman \& Ison, 1980).

Given the cross-modality nature of the phenomenon, and the fact that the reaction to an intense reflex-eliciting

This research was supported in part by grants from the US Environmental Protection Agency, CR-806820 and 812129. The statistical analyses were aided by NEI Center Support Grant to the Center for Visual Science and the University of Rochester, EY-01319. The authors are grateful to Lester Krueger and Walter Makous for their careful reading of this manuscript, and for their helpful comments. Reprint requests should be addressed to James R. Ison, Department of Psychology, Meliora Hall, University of Rochester, Rochester, NY 14627. stimulus can be inhibited by a near-threshold preliminary stimulus (e.g., Ison et al., 1986; Ison \& Pinckney, 1983; Reiter \& Ison, 1977), it is not surprising to find that reflex inhibition does not result from sensory masking of the eliciting stimulus by the lead stimulus. Thus, Ison and Krauter (1974) and Cohen, Hoffman, and Stitt (1981) have shown that the inhibitory effect of one stimulus on the response to a second stimulus is not reduced if a response to the first stimulus is itself inhibited by the introduction of a still earlier lead stimulus.

The magnitude of the inhibitory effect is determined largely by variation in, first, the temporal interval by which the preliminary stimulus leads the eliciting stimulus, and second, the intensity of the preliminary stimulus (Hoffman \& Ison, 1980). In addition, it has been shown that the inhibitory effects of two leading stimuli combine when they are presented in a stimulus compound just before reflex elicitation, either with their simultaneous presentation or with a brief gap intervening between the two components (Ison, Zuckerman, \& Russo, 1975). It can be expected, therefore, that if an acoustic prestimulus is delivered through earphones to both ears, it should provide more reflex inhibition than the same impulse delivered to one ear with silence in the other, on two grounds-first, because binaural stimuli are heard as being somewhat louder than monaural stimuli (Licklider, 1951), and second, because the binaural stimulus may be thought of as a compound consisting of two separate 
monaural components. However, the surprising outcome of experiments of this sort, which have been designed to study the effect of monaural or binaural acoustic input on the human blink reflex elicited by a cutaneous stimulus, is that the monaural stimulus provides more reflex inhibition (Marsh, Hoffman, \& Stitt, 1976). The phenomenon occurs across a wide range of stimulus intensities (Hoffman \& Stitt, 1980; Ison \& Pinckney, 1980), and even the offset of a monaural stimulus is more inhibitory than the offset of a binaural stimulus (Stitt, Hoffman, \& DeVido, 1980). The lesser impact of binaural presentation is not absolute but graduated according to the size of the interaural disparity. Thus, binaural input is as effective as monaural input, if the amplitude of the sound at one ear differs from the other by 20 to $40 \mathrm{~dB}$ (Hoffman \& Stitt, 1980).

One explanation of the particular effectiveness of the monaural stimulus is that the mechanisms that inhibit reflex expression are the targets of a comparator process that generates a signal proportional in strength to the difference between the inputs to the two ears. The output of the comparator mechanism, which is assumed to receive antagonistic input from the two ears, must be great enough to overcome a summation mechanism that receives supportive input from the two ears and is responsible for the loudness advantage of binaural presentation. In the prior experiments in which the greater inhibitory effect of monaural stimulus input on reflex expression has been demonstrated, the stimuli to the two ears were delivered simultaneously. In the present experiments, we examined whether the relative impact of the monaural stimulus would be reduced if the stimuli to the two ears were presented successively in time rather than together. The inhibitory effect of bilateral acoustic stimulation was measured as it varied with the imposition of different delays between the two successive inputs. Our intent in this research was to describe the temporal persistence of the antagonistic effect of one lateralized acoustic input on its contralateral counterpart.

\section{METHOD}

\section{Subjects}

The research participants were 24 undergraduate volunteers, 17 women and 7 men, 12 serving in each of two experiments. None had any known hearing or neurological problems.

\section{Apparatus}

The subjects sat comfortably in a barber shop chair housed within a double-walled sound-attenuating chamber (manufactured by the Industrial Acoustics Company, Inc., it was $3.7 \times 2.1 \times 2.4 \mathrm{~m}$ ). They were monitored with closed-circuit TV and an intercom system. The preliminary stimuli were noise pulses provided by a white noise source, gated through an electronic switch into a pair of matched TDH-39 earphones. Acoustic stimulus durations and interstimulus intervals were set by electronic timers. The intensity of these stimuli was measured with a General Radio Type 1561 sound-level meter and an earphone coupler, when the stimuli were set at long durations. An electric shock to the forehead elicited the eyeblink reflex. It was a bipolar square-wave shock, provided by a Grass biomedical stimulator (Model SD5) and a constant current unit (Model CCU), and it was delivered to the skin through two surface electrodes (Beckman miniature $\mathrm{Ag} / \mathrm{AgCl}$ ). The electrodes were fixed with adhesive disks vertical to the right pupil in forward gaze, $1 \mathrm{~cm}$ (cathode) and $2 \mathrm{~cm}$ above the brow. The site was identified as overlying the supraorbital branch of the trigeminal nerve by the subject's report that a probe threshold stimulus provided a tingling sensation across the scalp. Other electrodes of the same type were placed over the inferior orbicularis muscles of the right eye, at the lateral canthus and a medial position, to pick up eyeblink EMG activity. A ground electrode was located on the right temple, equidistant from the recording and stimulating pairs. Electrode impedance was less than $10 \mathrm{k} \Omega$. The EMG was fed through FET preamplifiers with half-amplitude bandpass from $30 \mathrm{~Hz}$ to $10 \mathrm{kHz}$, observed on a Tektronix storage CRT, and stored on magnetic tape for off-line analysis.

\section{Procedure}

The subjects first read a consent form that described the general procedures to be encountered in the experimental setting. Following their agreement to continue as participants, they were set up with surface stimulating and recording electrodes, according to standard procedures. They were given magazines to read during the experiment, but asked to attend first to the tactile forehead stimuli so that the experimenter could calibrate the apparatus and set the stimulus levels. The detection threshold for the forehead shock was determined by the ascending and descending method of limits, and then the threshold for the major EMG component of the cutaneous eyeblink was found (R2: cf. Sanes \& Ison, 1979) by the ascending method of limits. A current just sufficient to elicit the reflex on every trial was used to begin the experiment, and it was increased by 0.5 to $1.0 \mathrm{~mA}$ thereafter, if necessary, to maintain stable responding. Any necessary changes were made after all of the stimulus conditions had been presented an equal number of times at the lesser intensity. The mean intensity used in the experiment was $5.2 \mathrm{~mA}$ $(S D=2.3 \mathrm{~mA})$. The subjects never reported that the stimulus was painful. The acoustic stimuli that were used to inhibit the response were 90-dB noise pips which had a duration of $1 \mathrm{msec}$. No subject ever responded to these preliminary stimuli alone. The EMG activity elicited by the shock was rectified and summed over a 50msec period, beginning approximately $30 \mathrm{msec}$ after the eliciting stimulus, these numbers varying slightly with individual differences in response latency and duration.

Two experiments were run. In the first experiment, we examined the inhibitory effect of a monaural stimulus compared to a binaural stimulus and to bilateral stimuli that had different temporal delays between their two components. In the second experiment, we also compared the inhibitory effect of a monaural stimulus with that of a binaural stimulus, and we additionally varied the intrastimulus delay for bilateral stimulus presentation and for double-pip unilateral stimuli. In Experiment 1, six conditions were employed: (1) the baseline control condition-that is, a shock to the forehead presented alone with no preceding noise pips, given twice in each block of seven trials; (2) a monaural condition in which a single noise pip was delivered equally often to the left or to the right ear, in random sequence, 100 msec before the eliciting stimulus; (3) a binaural condition in which the same noise pip was delivered simultaneously to each ear, $100 \mathrm{msec}$ before the eliciting stimulus; and $(4,5$, and 6) three bilateral conditions in which the noise pips were delivered at different times, 1,4 , or $8 \mathrm{msec}$ apart, onset-to-onset, randomly left or right leading. In Experiment 2, seven conditions were used: (1) the baseline control condition, shock alone with no preceding noise pips; (2) a monaural condition in which a single noise pip was presented equally often to the left or right ear, $100 \mathrm{msec}$ before the eliciting stimulus; $(3,4$, and 5$)$ three unilateral conditions in which two noise pips were given to the same ear, equally often the left or right, 1, 4, or $8 \mathrm{msec}$ apart; (6) a binaural condition in which the noise pips were given simultaneously to the two ears; 
and (7) a bilateral condition in which two noise pips were given, first in one ear and then in the other, $8 \mathrm{msec}$ apart. In each of the double pulse conditions, the mean interval between the preliminary stimuli and the eliciting stimulus was $100 \mathrm{msec}$. (It should be noted that the small difference between conditions in the interval from the leading prepulse to the eliciting stimulus of, at maximum, 104 versus $100 \mathrm{msec}$, would be of no consequence.)

The order of trials for each subject followed separate $7 \times 7$ Latin squares in which each condition appeared once in each row and once in each column. Thus each subject received a total of 49 trials, at an approximate intertrial interval of $30 \mathrm{sec}$. For the analysis of the data, each subject's total integrated response was set at a value of 100 and then divided proportionately according to the respective mean values for the six or the seven different conditions. These numbers were subjected to analysis of variance (ANOVA) and a priori $t$ tests.

\section{RESULTS}

\section{Experiment 1}

Figure 1 depicts the mean relative response magnitudes and the standard errors of the mean for each of the six different conditions of Experiment 1. The ANOVA of these response amplitudes across all six conditions yielded a significant effect $[F(5,55)=15.04, p<.01]$. Three findings are obvious in these data: (1) Both monaural and binaural stimuli inhibited the cutaneous eyeblink reflex response $[t(11)=4.68$ (monaural), 3.22 (binaural), $p<.01$ ]; (2) inhibition was greater with the monaural stimulus $[t(11)=2.43, p<.05]$; (3) the bilateral stimulus became more effective with an increasing delay between its two components $[F(3,33)=6.18, p<.01]$, this following primarily a linear trend $[F(1,11)=17.52$, $p<.01]$. The difference between the $4 \mathrm{msec}$ and the $8 \mathrm{msec}$ conditions was reliable $[t(11)=2.25, p<.05]$,

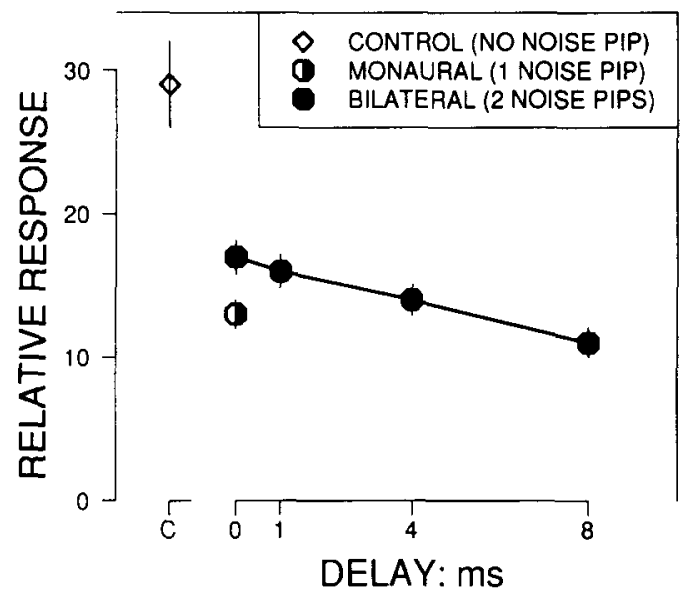

Figure 1. Relative mean eyeblink amplitudes (and their standard errors) elicited by a shock to the forehead, for the control condition (C) when the shock was given alone; and for conditions in which the shock was preceded by a monaural noise pip given 100 msec prior to the shock (depicted at $\mathbf{0}$ msec), or by bilateral noise pips varying in the time between successive inputs (depicted at $0,1,4$, or $8 \mathrm{msec}$ ). showing that the delay effect was not complete by $4 \mathrm{msec}$. However, reflex inhibition provided by the monaural stimulus was not reliably different from the inhibitory effect of bilateral stimuli with interpip delays of 4 or $8 \mathrm{msec}$.

These data reveal that an intrastimulus delay had an increasing positive influence on the bilateral stimulus, so that at the longer delays it was the equal of the monaural stimulus in inhibiting the reflex response to the subsequent cutaneous stimulus. However, it may not be appropriate to conclude that the mutual antagonism that exists between the two components of the bilateral stimulus had entirely dissipated at delay intervals of 4-8 msec. As described earlier, the inhibitory consequences of the two elements of a compound stimulus combine to determine the effect of the compound on reflex activity (Ison et al., 1975), and if the bilateral stimulus were perceived as a two-element compound, then their combined effect might cancel a continued superiority of unilateral over bilateral presentation. An unequivocal description of the temporal decay of contralateral antagonism would follow from a comparison of unilateral versus bilateral acoustic stimuli, in which both stimuli were composed of two-element compounds. In the second experiment, therefore, we compared the inhibitory effects on reflex expression of pairs of noise pips presented either unilaterally or bilaterally, with various delays imposed within the stimulus pair.

\section{Experiment 2}

Figure 2 depicts the mean relative response magnitudes and the standard errors for each of the seven conditions of the second experiment. The data confirmed the earlier findings, in showing that the monaural stimulus inhibited reflex expression more than the binaural stimulus did, and they further demonstrated that some difference between unilateral and bilateral stimuli remained for as long as $8 \mathrm{msec}$. An overall ANOVA showed that the conditions were reliably different $[F(6,66)=16.07, p<.01]$. An ANOVA performed just within the unilateral condition showed that the effectiveness of these stimuli was enhanced by an intrastimulus delay $[F(3,33)=3.96$, $p<.05]$, this following primarily a linear trend $(p<.01)$. It is noteworthy that the greater inhibitory effect of the 8-msec delayed pair compared to the 4-msec pair was of marginal significance $[t(11)=2.01$, $p<.07]$. The $2 \times 2$ factorial ANOVA performed on the corresponding points of the unilateral and bilateral presentations (the standard monaural versus binaural comparison, plus the two 8-msec delayed pairs) yielded an overall effect of mode of presentation $[F(1,11)=26.10$, $p<.01$ ]. It is especially important that unilateral presentation had a greater inhibitory effect on the subsequent cutaneous reflex even when $8 \mathrm{msec}$ separated the two components of the unilateral and the bilateral stimuli [ $t(11)$ $=2.65, p<.05]$. The slopes of the unilateral and bilateral functions differed marginally $[F(1,11)=4.60$, $p=.06]$, and when the curves were extrapolated, they converged on a delay time of about $15 \mathrm{msec}$. 


\section{DISCUSSION}

These experiments demonstrate again that a single noise presented in one ear, with silence in the other, inhibits a subsequent cutaneous reflex more than do two identical noises delivered simultaneously to both ears. They show further, as was expected on the hypothesis that inhibition is increased by an interaural disparity, that the relative disadvantage of the bilateral stimulus is reduced when the two ears receive their noise inputs one after the other, rather than simultaneously. Given the many demonstrations that reflex inhibition is graded according to the intensity of the leading stimulus (Hoffman \& Ison, 1980), and the fact that two leading stimuli have been shown to combine in their inhibitory effects (Ison et al., 1975), the greater inhibitory effect of the monaural stimuli suggests that the neural mechanisms responsible for reflex inhibition must receive an especially strong afferent signal when the acoustic event is heard in one ear and not the other. Hoffman and Stitt (1980) reported that a difference between the intensities of the stimuli presented simultaneously to the two ears enhanced the inhibitory effect of a binaural stimulus on reflex behavior. They proposed, therefore, that the control mechanisms that inhibit reflex output receive a substantial connection from a comparator process that calculates its output according to the interaural disparity between its inputs. The present data add to those of Hoffman and Stitt by demonstrating a comparable effect for bilateral stimuli with increasing interaural temporal disparity. A distinctive contribution of these data, however, is that the time course of the difference between the bilateral and unilateral modes of presentation is considerably different from that expected if the effective consequence of interaural disparity was a difference in the perceived spatial location of the acoustic stimuli.

A well-recognized neural representation of a bilateral comparator process is the set of cells in the auditory brainstem that receive excitatory inputs from one ear and antagonistic inhibitory inputs from the other. These cells function in a neural system that encodes spatial information from interaural differences in timing and intensity. Although at first blush it is plausible that activity within this system might be responsible both for the observations of Hoffman and Stitt (1980) and for the present data, we note that both the temporal and the intensity operating ranges of these bilateral cells as they support spatial localization are smaller by an order of magnitude than the interaural disparity necessary to effect differences in reflex inhibition. If this particular bilateral system does play a special role in reflex modification, it does not do so as any simple consequence of its classic perceptual function. Physical considerations (Fedderson, Sandel, Teas, \& Jeffress, 1957), electrophysiology (Phillips \& Brugge, 1985), and psychophysics (Gulick, 1971, pp. 188, 191) all agree that sound localization is computed on the basis of temporal differences of no more that $0.7 \mathrm{msec}$, and

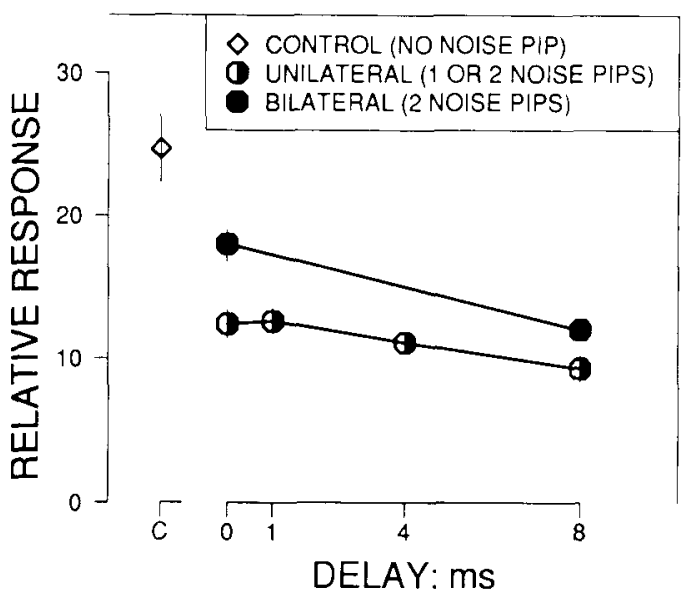

Figure 2. Relative mean eyeblink amplitudes (and their standard errors) elicited by a shock to the forehead, for the control condition (C) when the shock was given alone; and for conditions in which the shock was preceded by a single monaural noise pip given $100 \mathrm{msec}$ prior to the shock (depicted at 0 msec), or by two unilateral noise pips (depicted at 1, 4, or 8 msec), or two bilateral noise pips (depicted at 0 or $8 \mathrm{msec}$ ), each varying in the time between successive inputs.

amplitude differences of less than $10 \mathrm{~dB}$. But the experiments on reflex modification show that reduced efficacy of bilateral presentation in controlling reflex expression persists with interaural delays of at least $8 \mathrm{msec}$, and with interaural intensity disparities of up to $20-40 \mathrm{~dB}$.

The subtle interaural differences shown in physical, psychophysical, and electrophysiological experiments to be maximally effective for sound localization have no effect whatsoever on the reflex-inhibitory effect of bilateral acoustic input. Conversely, the profound interaural disparity necessary to alter the influence of the bilateral stimulus on reflex expression has no counterpart in the domain of sound localization. These conclusions are most evident in the contrast between the perceptual appearance of the stimulus arrays and their influence on reflex expression. (The experimenters were the observers for this brief study.) When two noise pips were presented bilaterally but simultaneously, a single auditory event was located in the center of the head, and when the pip in one ear led the other by just $1 \mathrm{msec}$, the sound shifted unequivocally to the leading ear: The perceived lateralization shift resulting from a 1-msec delay was $90^{\circ}$ for these stimuli, which agrees with the literature. Thus the change in spatial location reaches its maximum within $1 \mathrm{msec}$, but it will be noted from Figure 1 that a bilateral delay of $1 \mathrm{msec}$ is not accompanied by an enhancement in reflex expression. Speaking further to the difference between the interaural delays important in spatial location and those important for reflex inhibition is our finding in the second experiment that the difference between unilateral and bilateral modes of presentation remained significant even when a gap of $8 \mathrm{msec}$ separated the noise pips that made up each of the stimuli. Additional evidence that changes 
in spatial location and changes in reflex inhibition are not at all matched is given in our failure to perceive any further changes in sound location when the bilateral pips were separated by 4 and $8 \mathrm{msec}$, for these were the temporal delays shown to be necessary for altering reflex expression. A critical perceptual effect at these long delays is that the two pips were no longer fused. The stimulus arrays with internal gaps of 4 and $8 \mathrm{msec}$ gave rise to the perception of two distinct pips, both of which were at the same ear with unilateral presentation, at one ear and then the other with bilateral presentation.

In Experiment 2, it was shown that reflex inhibition increased with an increase in intrastimulus delay for unilateral as well as bilateral presentation. These data reveal that an auditory stimulus presented to one ear has a time-dependent ipsilateral antagonistic effect on a subsequent input to the same ear, in addition to the contralateral antagonism we hypothesize as being responsible for the disadvantage of binaural stimuli compared to monaural stimuli in inhibiting reflex expression. The difference between contralateral and ipsilateral inhibition is that the contralateral effect is more intense.

The several comparisons between the effects of different magnitudes of interaural variation on judgments of spatial location versus the degree of reflex inhibition produced by bilateral stimuli suggest that no simple homologue exists in the bilateral auditory system that can produce the differences in the reflex effects of monaural and binaural stimuli and also service the perceptual functions that are represented in spatial localization. Both types of data reveal the workings of strong antagonistic connections between the contralateral auditory pathways, but the differential effectiveness of unilateral versus bilateral stimuli in inhibiting reflex output results from a process that persists considerably longer than that presumed to be responsible for perceived differences in spatial localization: Reflex inhibition provides evidence for the presence of the bilateral comparator mechanism that could retain the identity of the leading ear for a prior acoustic input for at least $8 \mathrm{msec}$, and possibly as long as $15 \mathrm{msec}$.

\section{REFERENCES}

Cohen, M. E., Hoffman, H. S., \& StrtT, C. L. (1981). Sensory magnitude estimation in the context of reflex modification. Journal of Experimental Psychology: Human Perception \& Performance, 7, 1363-1370.

Cranney, J., Cohen, M. E. (1985). The glabella startle reflex: Inhibition by frequency and intensity modulations. Perception \& Psychophysics, 37, 28-34.

Fedderson, W. C., Sandel, T. T., Teas, D. C., \& Jeffress, L. A. (1957). Localization of high-frequency tones. Journal of the Acoustical Society of America, 29, 988-991.
Graham, F. K., Putnam, L. E., Leavitt, L. A. (1975). Lead stimulation effects on human cardiac orienting and blink reflexes. Journal of Experimental Psychology: Human Perception \& Performance, 1, 161-169.

Gulick, W. L. (1971). Hearing. New York: Oxford University Press. Hulgard, E. R. (1933). Reinforcement and inhibition of eyelid reflexes. Journal of General Psychology, 8, 85-111.

Hoffman, H. S., Ison, J. R. (1980). Reflex modification in the domain of startle: I. Some empirical findings and their implications for how the nervous system processes sensory input. Psychological Review, 87, 175-189.

Hofrman, H. S., \& STITT, C. L. (1980). Inhibition of the glabella reflex by monaural and binaural stimulation. Joumal of Experimental Psychology: Human Perception \& Performance, 6, 769-776.

Ison, J. R., Foss, J. A., Falcone, P., Sakovits, L., Adelson, A. A., Burton, R. I. (1986). Reflex modification: A method for assessing cutaneous dysfunction. Perception \& Psychophysics, 40, 164-170.

Ison, J. R., Horfman, H. S. (1983). Reflex modification in the domain of startle: II. The anomalous history of a robust and ubiquitous phenomenon. Psychological Bulletin, 94, 3-17.

IsON, J. R., KRAUTER, E. E. (1974). Reflex inhibiting stimuli and the refractory period of the acoustic startle reflex in the rat. Journal of Comparative \& Physiological Psychology, 89, 39-49.

IsoN, J. R., PINCKNEY, L. [A]. (1980). Reflex modulation in humans by monaural and binaural auditory stimulation. Bulletin of the Psychonomic Society, 15, 285-287.

Ison, J. R., \& PINCKNeY, L. A. (1983). Reflex inhibition in humans: Sensitivity to brief silent periods in white noise. Perception \& Psychophysics, 34, 84-88.

Ison, J. R., Zuckerman, M., \& Russo, J. M. (1975). Combination rules for inhibitory stimuli. Joumal of Experimental Psychology: Animal Behavior Processes, 1, 318-325.

Krauter, E. E., Leonard, D. W., \& Ison, J. R. (1973). Inhibition of the human eyeblink by a brief acoustic stimulus. Journal of Comparative \& Physiological Psychology, 84, 246-251.

LICKLIDER, J. C. R. (1951). Basic correlates of the auditory stimulus. In S. S. Stevens (Ed.), Handbook of Experimental Psychology (pp. 985-1039). New York: Wiley.

MARSh, R. R., HofFman, H. S., StitT, C. L. (1976). Eyeblink inhibition by monaural and binaural stimuli: One ear is better than two. Science, 192, 390-391.

Phillips, D. P., \& Brugge, J. F. (1985). Progress in neurophysiology of sound localization. In M. R. Rosenzweig \& L. W. Porter, (Eds.), Annual Review of Psychology, 36, 245-274.

ReITER, L. A., Ison, J. R. (1977). Inhibition of the human eyeblink reflex: An examination of the Wendt-Yerkes method for threshold detection. Joumal of Experimental Psychology: Human Perception \& Performance, 3, 325-336.

SANES, J. N., \& IsON, J. R. (1979). Conditioning auditory stimuli and the cutaneous eyeblink in humans: Differential effects according to oligosynaptic or polysynaptic central pathways. Electroencephalography \& Clinical Neurophysiology, 47, 546-555.

StitT, C. L., Hoffman, H. S., \& DeVIDo, C. J. (1980). Modification of the human glabella reflex by antecedent acoustic stimulation. Perception \& Psychophysics, 27, 82-88.

(Manuscript received March 13, 1989; revision accepted for publication October 10, 1989.) 